Article

\title{
Higher Diet Quality is Associated with Lower Odds of Low Hand Grip Strength in the Korean Elderly Population
}

\author{
Hyesook Kim $®$ and Oran Kwon * \\ Department of Nutritional Science and Food Management, Ewha Womans University, 52, Ewhayeodae-gil, \\ Seodaemun-gu, Seoul 03760, Korea \\ * Correspondence: orank@ewha.ac.kr; Tel.: +82-2-3277-6860; Fax: +82-2-3277-6860
}

Received: 22 May 2019; Accepted: 27 June 2019; Published: 29 June 2019

\begin{abstract}
Single nutrients or food groups have been associated with physical performance. However, little is known about the association of overall diet quality with hand grip strength (HGS), a predictive parameter in the prognosis of chronic disease morbidity and mortality, or quality of life. This study examined the association between HGS and three indices- the Korean Healthy Eating Index (KHEI), the Alternate Mediterranean Diet (aMED), and Dietary Approaches to Stop Hypertension (DASH) - using data obtained on Korean elderly persons aged $\geq 65$ years $(n=3675)$ from a nationally representative database. The cross-sectional data was collected as part of the Korea National Health and Nutrition Examination Survey (KNHANES, 2014-2016). Dietary intake data from the 24-h recall method were used to calculate diet quality scores. The cutoff value for low HGS was defined as the value corresponding to the lowest 20th percentile of HGS of the study population (men, $28.6 \mathrm{~kg}$; women, $16.5 \mathrm{~kg}$ ). Higher index scores for diet quality were associated with $32 \%-53 \%$ lower odds of low HGS. Better overall diet quality may be associated with higher HGS in the elderly Korean population.
\end{abstract}

Keywords: dietary quality; muscle strength; KNHANES

\section{Introduction}

Aging is accompanied by widespread and typical changes in the human body. The most prominent physical change that occurs during the aging process is the loss of muscle mass, resulting in decreased muscle strength [1]. Reduced muscle strength is linked to a loss of independence and an increased risk of reduced mobility, disability, and mortality [2]. Muscle strength is one of the most decisive indicators of health status in older adults [3], and therefore, maintaining muscle strength is very important for reducing functional limitations in older adults.

Hand grip strength (HGS) is recognized as a general indicator of overall muscular strength [4], as well as an important biomarker of health and disease status [5]. HGS has been reported to be associated with various chronic diseases, including cardiovascular disease, diabetes, and metabolic syndrome [6-9]. Moreover, the predictive validity of HGS for decline in cognition, mobility, and mortality was established in a recent systematic review and meta-analysis [10].

HGS can be influenced by many factors, such as age [11], gender [12], race [13], and exercise [14]. Nutritional status is one of the major determinants of HGS with aging. Many studies have reported a link between HGS and intake of specific single nutrients, including protein [15], vitamin D [16], and antioxidants, like carotenoids [17], vitamin E [18], vitamin C [19], and selenium [20]. However, recent dietary research has focused on the whole diet, rather than single nutrients or individual food groups, because dietary components are consumed in combination and interact with one 
another. There is some evidence relating HGS to overall dietary quality indicators, such as the Healthy Eating Index (HEI) [15,21], Mediterranean Diet (MED) [22-24], Dietary Approaches to Stop Hypertension (DASH) [15], and Nordic Diet Score (NDS) [25], from European [22-25] and North American studies [15,21].

South Korea has one of the most rapidly aging populations in the world [26], and age-related poor muscle strength is becoming a major public health problem. Thus, more research on the association between overall diet quality and HGS, an important index of low muscle strength, is needed in the Korean population. While many studies have been conducted in Western countries, Jeong's work [27] is the only research utilizing Korean subjects. Recently, Jeong et al. [27] reported on the positive relationship between the recommended food score (RFS), one of the indicators of overall diet quality, and HGS in elderly Korean women participating in the 2014-2015 National Fitness Award project. The Korean Ministry of Health and Welfare has begun to include the HGS measurement in the population-based Korea National Health and Nutrition Examination Survey (KNHANES) in 2014. Since then, research has been published on the relationship between HGS and some health outcomes, such as hypertension [28], chronic obstructive pulmonary disease [29], and quality of life [30,31]. However, to the best of our knowledge, no studies have reported on the relationship between overall diet quality and HGS among a representative Korean population.

Therefore, this study was conducted to investigate the association between diet quality indices-the Korean Healthy Eating Index (KHEI), the alternate Mediterranean Diet (aMED), and DASH—and HGS in the Korean elderly, using the nationally representative KNHANES data.

\section{Materials and Methods}

\subsection{Study Population}

This study was based on data from the second and third years (2014-2015) of the KNHANES VI (2013-2015) and the first year (2016) of KNHANES VII (2016-2018), conducted by the Korea Centers for Disease Control and Prevention (KCDC). The KNHANES employs multi-stage stratified cluster sampling for the selection of household units among non-institutionalized residents in the Republic of Korea. The survey consists of a health interview, a health examination, and a nutrition assessment. This study was approved by the Institutional Review Board of the KCDC, and written informed consent was obtained from all subjects. Detailed information about the survey is available on the website [32] (http://knhanes.cdc.go.kr).

Among the participants, 4766 individuals ( 2077 men and 2689 women) aged $\geq 65$ years participated in the survey. We excluded those with missing values for HGS (207 men and 509 women) and 24-h dietary recall (149 men and 167 women). Subjects (12 men and 47 women) with implausible energy intakes of $<500 \mathrm{kcal}$ or $>8000 \mathrm{kcal}$ were also excluded. The final sample for the analysis comprised of 3675 individuals (1709 men and 1966 women).

\subsection{General Characteristics}

The health interview and health examination portions of the survey were used to obtain the demographic and socioeconomic characteristics of the participants, including age, health status, body mass index (BMI), education level, smoking status, alcohol consumption, physical activity, and age at menarche.

Health status included physician-diagnosed chronic conditions (hypertension, hyperlipidemia, stroke, myocardial infarction or angina, arthritis, asthma, diabetes mellitus, cancer, depression, renal failure, and chronic obstructive pulmonary disease). BMI was calculated as weight $(\mathrm{kg}) /(\text { height }(\mathrm{m}))^{2}$. Education level was categorized as elementary school or less versus more than elementary school. Smoking status was classified into three categories (non-smoker, ex-smoker, and current smoker). Alcohol consumption was classified into five categories (never, $<1$ drink/month, 1 drink/month, 2-4 drinks/month or $\geq 4$ drinks/month within the last year). Physical activity was divided into five 
categories according to the number of days when strength exercise occurred within week prior (none, 1 day/week, 2 days/week, 3 days/week, 4 days/week or $\geq 5$ days/week). For women, the age at menarche was recorded.

\subsection{Dietary Assessment}

KNHANES conducts a 24-h recall survey for all participants aged over 12 months (1-80 years of age), whereas the food frequency questionnaire is conducted only for adults aged 19-64 years. Therefore, we used data from a single 24-h recall period to investigate the dietary intake of older adults aged over 65 years. Participants reported all foods and drinks consumed during the previous day in face-to-face interviews.

Table 1 identifies the components and standards for optimal scoring in the diet quality indices, such as KHEI, aMED, and DASH; specific details are described below.

\subsubsection{KHEI}

The KHEI was developed [33] to quantify adherence to dietary guidelines for Korean adults [34], the 2010 Dietary Reference Intakes for Koreans [35], and the objectives of the Health Plan 2020 [36]. The KHEI scores 14 components for a maximum total of 100 points. Eight components—whole grains, refined grains, fruit intake (excluding juice), fruit intake (including juice), vegetable intake (excluding Kimchi and pickles), vegetable intake (including Kimchi or pickles), ratio of white meat-to-red meat, and percentages of energy from carbohydrates_-are worth 0-5 points; six components-breakfast, milk and dairy, protein foods, sodium, percentages of energy from empty calorie foods, and percentages of energy from fat - are worth 0-10 points.

\subsection{2. aMED Score}

The first Mediterranean diet score, developed by Trichopoulou et al. [37], takes into account the scientific literature on diet and chronic disease risk. The score we used, aMED, was modified by Fung et al. [38]. The aMED scores nine components for a total of 9 points. The intakes of nine food groups were dichotomized using sex-specific median values as the cutoff. A score of 1 was assigned for consumption above the median level of presumed beneficial foods (whole grains, vegetables (excluding potatoes), fruit (including juice), nuts, legumes, fish, and the ratio of monounsaturated fatty acids-to-saturated fatty acids) and for consumption below the median level of presumed detrimental foods (red and processed meat), and a score of 0 was assigned for all other. For alcohol, 1 point was assigned to men who consumed between 10 and $25 \mathrm{~g} /$ day and to women who consumed between 5 and $15 \mathrm{~g} /$ day, versus a score of 0 .

\subsubsection{DASH Score}

The DASH score was developed by Fung et al. [39] based on foods and nutrients emphasized or minimized in the DASH diet, according to an eating guide developed by the National Heart, Lung and Blood Institute [40]. DASH scores eight components (seven food groups and one nutrient)—each worth 5 points-for a maximum total of 40 points. The scoring system is based on sex-specific quintile rankings within the study population. Points range from 5 (highest quintile) to 1 (lowest quintile) for whole grains, vegetables (excluding potatoes), fruit (including juice), nuts and legumes, and low-fat dairy, and from 1 (highest quintile) to 5 (lowest quintile) for sugar-sweetened beverages, red and processed meat, and sodium. Among these eight components, the survey of low-fat dairy food intake was not performed in the 24-h recall portion of KNHANES. Therefore, in this study, low-fat dairy was used as a whole dairy product. 


\subsection{Hand Grip Strength}

HGS was measured three times in each hand, using a digital hand dynamometer (digital grip strength dynamometer, TKK-5401, Takei Scientific Instruments Co., Ltd., Tokyo, Japan). Trained medical technicians instructed the seated subjects to hold the dynamometer with the second finger nodes of the working hand at an angle of $90^{\circ}$ to the handle and to squeeze the handle as firmly as they could. After subjects slowly stood up, HGS was measured during expiration. Between each measurement, a $60 \mathrm{~s}$ resting interval was allowed. The HGS value used in the analysis was the highest of the six measured values [41].

\subsection{Statistical analysis}

All statistical analyses were performed using SAS software version 9.4 (SAS Institute, Cary, NC, USA). Due to the complex sampling design of the KNHANES study, the relevant primary sampling units, stratification, and sample weights were considered in our analysis. For descriptive statistics, continuous variables are expressed as weighted means and SEMs, and categorical variables are expressed as numbers and weighted percentages, using the procedures of SURVEYMEANS and SURVEYFREQ, respectively. SURVEY LOGISTIC analysis was performed to estimate the odds ratios (ORs) and 95\% confidence intervals (CIs) for risk of low muscle strength across tertiles of each diet quality index, with the 1st tertile set as the reference. Model 1 was adjusted for age (years), number of physician-diagnosed chronic conditions (n), BMI $\left(\mathrm{kg} / \mathrm{m}^{2}\right)$, education (selementary school, $>$ elementary school), smoking (non-smoker, ex-smoker, or current smoker), alcohol consumption (never, $<1$ drink/month, 1 drink/month, 2-4 drinks/month or $\geq 4$ drinks/month), energy intake (kcal), and age at menarche (women only) (years). Model 2 was adjusted for the variables in model 1, plus physical activity assessed by days of strength exercise within the last week (never, 1 day/week, 2 days/week, 3 days/week, 4 days/week or $\geq 5$ days/week). All reported probability tests were two-sided, and differences were considered significant at the $5 \%$ level.

\section{Results}

\subsection{General Characteristics}

Low muscle strength was defined as the lower 20th percentile of HGS (men, $28.6 \mathrm{~kg}$; women, $16.4 \mathrm{~kg})$, and 344 men (19.5\%) and 388 women (19.7\%) were classified into the low muscle strength group. Table 2 shows the characteristics of the men and women in the highest tertile (3rd tertile; most optimal diet quality) compared with the lowest tertile (1st tertile; poorest diet quality) for each diet quality index. Across all indices, both men and women in the 3rd tertile were more likely to be more educated, never to have smoked, be more physically active, and consume dietary supplements. Across all indices, men in the 3rd tertile had a high BMI; in contrast, there was no difference in the BMI across the tertile groups among women. Across all indices, women in the 3rd tertile were more likely to be younger, have higher energy intakes, and a younger age at menarche. For DASH, men in the 3rd tertile had a higher number of physician-diagnosed chronic conditions; in contrast, women in the 3rd tertile had a lower number of physician-diagnosed chronic conditions.

\subsection{Association of Diet Quality with Grip Strength}

Correlations among the total scores for all pairs of indices are presented in Table 3. For men, the correlations ranged from 0.38 (for KHEI and aMED) to 0.59 (for aMED and DASH). For women, the correlations ranged from 0.43 (for KHEI and aMED) to 0.60 (for aMED and DASH). All correlations were significant $(p<0.0001)$. 
Table 1. Scoring standards for each component of the KHEI, aMED, and DASH score ${ }^{1 .}$

\begin{tabular}{|c|c|c|c|c|c|c|c|c|c|}
\hline \multirow[b]{2}{*}{ Component } & \multicolumn{3}{|c|}{ KHEI $^{2}$} & \multicolumn{3}{|c|}{ aMED $^{3}$} & \multicolumn{3}{|c|}{ DASH $^{4}$} \\
\hline & $\begin{array}{l}\text { Maximum } \\
\text { Score }\end{array}$ & $\begin{array}{c}\text { Criteria for } \\
\text { Maximum Score }\end{array}$ & $\begin{array}{c}\text { Criteria for } \\
\text { Minimum Score }\end{array}$ & $\begin{array}{l}\text { Maximum } \\
\text { Score }\end{array}$ & $\begin{array}{c}\text { Criteria for } \\
\text { Maximum Score }\end{array}$ & $\begin{array}{l}\text { Criteria for Minimum } \\
\text { Score }\end{array}$ & $\begin{array}{l}\text { Maximum } \\
\text { Score }\end{array}$ & $\begin{array}{c}\text { Criteria for } \\
\text { Maximum Score }\end{array}$ & $\begin{array}{c}\text { Criteria for } \\
\text { Minimum Score }\end{array}$ \\
\hline Breakfast & 10 & $\begin{array}{c}\text { Have breakfast in } 2 \\
\mathrm{~d}\end{array}$ & Nothing & - & - & - & - & - & - \\
\hline Whole grains & 5 & $\geq 1 \mathrm{svg} / \mathrm{d}$ & $0 \mathrm{svg} / \mathrm{d}$ & 1 & Median or greater & Less than median & 5 & Highest quintile & Lowest quintile \\
\hline Refined grains & 5 & $\begin{array}{l}\text { Male: } \leq 4 \mathrm{svg} / \mathrm{d} \\
\text { Female: } \leq 3 \mathrm{svg} / \mathrm{d}\end{array}$ & $\begin{array}{l}\text { Male: } \geq 5 \mathrm{svg} / \mathrm{d} \\
\text { Female: } \geq 4 \mathrm{svg} / \mathrm{d}\end{array}$ & - & - & - & - & - & - \\
\hline Nuts and legumes & & & & & & & 5 & Highest quintile & Lowest quintile \\
\hline Nuts & - & - & - & 1 & Median or greater & Less than median & - & - & - \\
\hline Legumes & - & - & - & 1 & Median or greater & Less than median & - & - & - \\
\hline Fruits including juice & 5 & $\begin{array}{l}\text { Male: } \geq 3 \mathrm{svg} / \mathrm{d} \\
\text { Female: } \geq 2 \mathrm{svg} / \mathrm{d}\end{array}$ & $0 \mathrm{svg} / \mathrm{d}$ & 1 & Median or greater & Less than median & 5 & Highest quintile & Lowest quintile \\
\hline Fruit excluding juice & 5 & $\begin{array}{l}\text { Male: } \geq 1.5 \mathrm{svg} / \mathrm{d}, \\
\text { Female: } \geq 1 \mathrm{svg} / \mathrm{d}\end{array}$ & $0 \mathrm{svg} / \mathrm{d}$ & - & - & - & - & - & - \\
\hline $\begin{array}{l}\text { Vegetables including } \\
\text { Kimchi or pickles }\end{array}$ & 5 & $\geq 7 \mathrm{svg} / \mathrm{d}$ & $0 \mathrm{svg} / \mathrm{d}$ & 1 & Median or greater & Less than median & 5 & Highest quintile & Lowest quintile \\
\hline $\begin{array}{l}\text { Vegetables excluding } \\
\text { Kimchi and pickles }\end{array}$ & 5 & $\geq 4 \mathrm{svg} / \mathrm{d}$ & $0 \mathrm{svg} / \mathrm{d}$ & - & - & - & - & - & - \\
\hline Milk and dairy & 10 & $\geq 1 \mathrm{svg} / \mathrm{d}$ & $0 \mathrm{svg} / \mathrm{d}$ & - & - & - & 5 & Highest quintile & Lowest quintile \\
\hline Protein foods & 10 & $\begin{array}{l}\text { Male: } \geq 5 \mathrm{svg} / \mathrm{d} \\
\text { Female: } \geq 4 \mathrm{svg} / \mathrm{d}\end{array}$ & $0 \mathrm{svg} / \mathrm{d}$ & - & - & - & - & - & - \\
\hline Red and processed meat & - & - & - & 1 & Median or greater & Less than median & 5 & Lowest quintile & Highest quintile \\
\hline $\begin{array}{l}\text { Ratio of white meat to } \\
\text { red meat }\end{array}$ & 5 & 4: $1(\geq 4)$ & $\begin{array}{l}0 \mathrm{svg} / \mathrm{d} \text { (Red meat } \\
\text { only) }\end{array}$ & - & - & - & - & - & - \\
\hline Fish & - & - & - & 1 & Median or greater & Less than median & - & - & - \\
\hline $\begin{array}{l}\text { Sugar-sweetened } \\
\text { beverages }\end{array}$ & - & - & - & - & - & - & 5 & Lowest quintile & Highest quintile \\
\hline Alcohol & - & - & - & 1 & $\begin{array}{l}\text { Male: } 10-25 \mathrm{~g} / \mathrm{d} \\
\text { Female: } 5-15 \mathrm{~g} / \mathrm{d}\end{array}$ & $\begin{array}{l}\text { Male: }<10 \text { or }>25 \mathrm{~g} / \mathrm{d} \\
\text { Female: }<5 \text { or }>15 \mathrm{~g} / \mathrm{d}\end{array}$ & - & - & - \\
\hline Sodium & 10 & $\leq 2000 \mathrm{mg} / \mathrm{d}$ & $>85$ percentile & - & - & - & 5 & Lowest quintile & Highest quintile \\
\hline $\begin{array}{l}\text { Percentages of energy } \\
\text { from empty calorie } \\
\text { foods }\end{array}$ & 10 & $\leq 5 \%$ energy & $\geq 10 \%$ energy & - & - & - & - & - & - \\
\hline $\begin{array}{l}\text { Percentages of energy } \\
\text { from carbohydrates }\end{array}$ & 5 & $55 \sim 70 \%$ & $\begin{array}{l}<15 \text { percentile } \\
>85 \text { percentile }\end{array}$ & - & - & - & - & - & - \\
\hline $\begin{array}{l}\text { Percentages of energy } \\
\text { from fat }\end{array}$ & 10 & $15 \sim 25 \%$ & $\begin{array}{l}<15 \text { percentile } \\
>85 \text { percentile }\end{array}$ & - & - & - & - & - & - \\
\hline MUFA: SFA ratio & - & - & - & 1 & Median or greater & Less than median & - & - & - \\
\hline
\end{tabular}

${ }^{1}$ Scoring standards are based on one serving size from the Food nutritional value table easy to understand by consumers in one serving by National Academy of Agricultural Science ; KHEI Korean Healthy Eating Index; aMED, alternate Mediterranean Diet; DASH, Dietary Approaches to Stop Hypertension; svg, serving; d, day; -, not applicable; MUFA, Monounsaturated Fatty Acid; SFA, Saturated Fatty Acid. ${ }^{2}$ KHEI: 100 points total; 14 components: $5-10$ points each. ${ }^{3}$ aMED: 9 points total; 9 components: 1 point each. ${ }^{4}$ DASH: 40 points total; 8 components: 5 points each. 
Table 2. Descriptive characteristics of elderly men and women in the 2014-2016 KNHANES based on tertiles of the KHEI, aMED, and DASH score 1.

\begin{tabular}{|c|c|c|c|c|c|c|c|c|c|c|c|c|c|c|c|c|c|c|}
\hline & \multicolumn{6}{|c|}{ KHEI } & \multicolumn{6}{|c|}{ aMED } & \multicolumn{6}{|c|}{ DASH } \\
\hline & T1 & T2 & T3 & $p$-Value & $\begin{array}{l}p \text {-Value } \\
\text { (T1 vs. } \\
\text { T3) }\end{array}$ & $\begin{array}{l}p \text {-Value } \\
\text { (T2 vs. } \\
\text { T3) }\end{array}$ & T1 & T2 & T3 & $p$-Value & $\begin{array}{l}p \text {-Value } \\
\text { (T1 vs. } \\
\text { T3) }\end{array}$ & $\begin{array}{c}p \text {-Value } \\
\text { (T2 vs. } \\
\text { T3) }\end{array}$ & T1 & T2 & T3 & $p$-Value & $\begin{array}{l}p \text {-Value } \\
\text { (T1 vs. } \\
\text { T3) }\end{array}$ & $\begin{array}{l}p \text {-Value } \\
\text { (T2 vs. } \\
\text { T3) }\end{array}$ \\
\hline \multicolumn{19}{|l|}{ Men } \\
\hline$n$ & 569 & 570 & 570 & & & & 632 & 373 & 704 & & & & 594 & 489 & 626 & & & \\
\hline Low muscle strength ${ }^{2}, \mathrm{n}(\%)$ & $143(24.0)$ & $121(21.5)$ & $80(13.0)$ & $<0.001$ & & & $174(27.4)$ & $75(20.4)$ & $95(12.5)$ & $<0.001$ & & & $131(21.7)$ & $111(22.0)$ & $102(15.5)$ & 0.018 & & \\
\hline Age, $\mathrm{y}$ & $72.9 \pm 0.2$ & $72.1 \pm 0.2$ & $72.0 \pm 0.2$ & 0.007 & 0.004 & 0.689 & $73.3 \pm 0.2$ & $72.4 \pm 0.3$ & $71.5 \pm 0.2$ & $<0.001$ & $<0.001$ & 0.012 & $72.2 \pm 0.2$ & $72.8 \pm 0.3$ & $72.1 \pm 0.2$ & 0.080 & 0.582 & 0.030 \\
\hline BMI, $\mathrm{kg} / \mathrm{m}^{2}$ & $23.3 \pm 0.2$ & $23.8 \pm 0.1$ & $23.9 \pm 0.1$ & 0.008 & 0.005 & 0.907 & $23.4 \pm 0.2$ & $23.8 \pm 0.2$ & $23.8 \pm 0.1$ & 0.036 & 0.015 & 0.971 & $23.5 \pm 0.2$ & $23.5 \pm 0.1$ & $24.0 \pm 0.1$ & 0.038 & 0.028 & 0.030 \\
\hline Education, $n(\%)$ & & & & & & & & & & & & & & & & & & \\
\hline$<=$ Elementary school & $284(56.1)$ & $220(42.7)$ & $141(26.6)$ & $<0.001$ & & & $293(53.9)$ & $141(39.3)$ & $211(32.4)$ & $<0.001$ & & & $261(49.5)$ & $188(42.7)$ & $196(33.5)$ & $<0.001$ & & \\
\hline$>$ Elementary school & 219 (43.9) & $308(57.3)$ & $392(73.4)$ & & & & $271(46.1)$ & $207(60.7)$ & $441(67.6)$ & & & & $272(50.5)$ & $254(57.3)$ & $393(66.5)$ & & & \\
\hline No response & 66 & 42 & 37 & & & & 68 & 25 & 52 & & & & 61 & 47 & 37 & & & \\
\hline \multicolumn{19}{|l|}{ Smoking status, $n(\%)$} \\
\hline Nonsmoker & $96(18.0)$ & $96(16.0)$ & $139(27.1)$ & $<0.001$ & & & $113(19.7)$ & $68(17.6)$ & $150(22.3)$ & 0.060 & & & $93(16.4)$ & $99(20.5)$ & $139(23.8)$ & $<0.001$ & & \\
\hline Ex-smoker & $298(58.5)$ & $343(63.3)$ & $340(59.7)$ & & & & $333(57.3)$ & $228(63.9)$ & $420(61.6)$ & & & & $329(59.2)$ & $271(59.0)$ & $381(63.2)$ & & & \\
\hline Current smoker & $122(23.6)$ & $111(20.7)$ & $69(13.2)$ & & & & $135(23.1)$ & $61(18.5)$ & $106(16.1)$ & & & & 139 (24.4) & $86(20.5)$ & $77(13.0)$ & & & \\
\hline No response & 53 & 20 & 22 & & & & 51 & 16 & 28 & & & & 33 & 33 & 29 & & & \\
\hline \multicolumn{19}{|l|}{ Alcohol consumption, $n(\%)$} \\
\hline Never & $154(30.3)$ & $189(32.4)$ & $201(37.6)$ & $<0.001$ & & & $218(39.0)$ & $109(30.1)$ & $217(30.7)$ & 0.057 & & & $187(33.4)$ & $145(31.3)$ & $212(35.2)$ & 0.362 & & \\
\hline$<1$ Months & $57(10.4)$ & $83(15.9)$ & $110(19.7)$ & & & & $87(14.4)$ & $59(16.3)$ & $104(15.9)$ & & & & $80(13.3)$ & $71(16.2)$ & $99(16.9)$ & & & \\
\hline 1 Months & 75 (13.4) & $108(19.8)$ & $114(20.2)$ & & & & $90(15.1)$ & $78(22.0)$ & $129(18.1)$ & & & & $93(17.4)$ & $88(17.5)$ & $116(18.8)$ & & & \\
\hline 2-4 Months & $96(19.8)$ & $89(17.0)$ & $76(13.8)$ & & & & $81(14.5)$ & $53(15.5)$ & $127(19.4)$ & & & & $93(17.1)$ & $81(19.7)$ & $87(14.3)$ & & & \\
\hline$>=4$ Months & $136(26.1)$ & $82(14.8)$ & $47(8.7)$ & & & & $105(17.0)$ & $59(16.1)$ & $101(15.8)$ & & & & $110(18.8)$ & $72(15.3)$ & $83(14.8)$ & & & \\
\hline No response & 51 & 19 & 22 & & & & 51 & 15 & 26 & & & & 31 & 32 & 29 & & & \\
\hline \multicolumn{19}{|c|}{ Days of strength exercise (days/week), $n$ (\%) } \\
\hline No & $403(79.6)$ & $363(66.4)$ & $347(65.9)$ & $<0.001$ & & & $449(78.8)$ & $255(73.2)$ & $409(61.9)$ & $<0.001$ & & & $415(76.7)$ & 330 (73.9) & $368(62.1)$ & $<0.001$ & & \\
\hline 1 days/week & $6(1.1)$ & $11(2.0)$ & $6(0.9)$ & & & & $11(1.7)$ & $1(0.3)$ & $11(1.6)$ & & & & $8(1.7)$ & $9(1.8)$ & $6(0.7)$ & & & \\
\hline 2 days/week & $15(3.3)$ & $25(4.5)$ & $27(5.7)$ & & & & $17(3.2)$ & $14(3.5)$ & $36(6.1)$ & & & & $21(3.8)$ & $15(3.9)$ & $31(5.6)$ & & & \\
\hline 3 days/week & $16(3.2)$ & $20(4.2)$ & $24(4.1)$ & & & & $15(2.8)$ & $15(4.1)$ & $30(4.5)$ & & & & $16(3.0)$ & $13(2.7)$ & $31(5.5)$ & & & \\
\hline 4 days/week & $8(1.3)$ & $8(1.8)$ & $20(3.9)$ & & & & $8(1.3)$ & $11(3.5)$ & $17(2.6)$ & & & & $10(2.1)$ & $8(2.0)$ & $18(2.9)$ & & & \\
\hline$>=5$ days/week & $58(11.4)$ & $101(21.2)$ & $109(19.5)$ & & & & $65(12.1)$ & $54(15.4)$ & $149(23.2)$ & & & & $65(12.8)$ & $68(15.8)$ & $135(23.2)$ & & & \\
\hline \multirow{2}{*}{\multicolumn{19}{|c|}{$\begin{array}{l}\text { No response } \\
\text { Dietary supplement use, } n(\%)\end{array}$}} \\
\hline & & & & & & & & & & & & & & & & & & \\
\hline No & $385(68.2)$ & $342(59.0)$ & $295(49.8)$ & $<0.001$ & & & $433(69.1)$ & $218(57.2)$ & $371(51.5)$ & $<0.001$ & & & $392(65.2)$ & $296(60.1)$ & $334(52.4)$ & $<0.001$ & & \\
\hline Yes & $184(31.8)$ & $228(41.0)$ & $275(50.2)$ & & & & $199(30.9)$ & $155(42.8)$ & $333(48.5)$ & & & & $202(34.8)$ & $193(39.9)$ & 292 (47.6) & & & \\
\hline Energy intake, kcal/d & $\begin{array}{l}1916.4 \pm \\
37.3\end{array}$ & $\begin{array}{l}1969.8 \pm \\
35.1\end{array}$ & $\begin{array}{l}2002.8 \pm \\
33.67\end{array}$ & 0.209 & 0.078 & 0.482 & $\begin{array}{c}1746.6 \pm \\
32.5\end{array}$ & $\begin{array}{c}1934.3 \pm \\
41.3\end{array}$ & $\begin{array}{r}2161.4 \pm \\
30.2\end{array}$ & $<0.001$ & $<0.001$ & $<0.001$ & $\begin{array}{l}1917.4 \pm \\
33.8\end{array}$ & $\begin{array}{c}1981.7 \pm \\
42.6\end{array}$ & $\begin{array}{l}1991.4 \pm \\
32.1\end{array}$ & 0.222 & 0.105 & 0.856 \\
\hline $\begin{array}{l}\text { Number of physician diagnosed } \\
\text { chronic conditions }\end{array}$ & $1.3 \pm 0.1$ & $1.3 \pm 0.1$ & $1.4 \pm 0.1$ & 0.407 & 0.246 & 0.232 & $1.2 \pm 0.1$ & $1.4 \pm 0.1$ & $1.3 \pm 0.1$ & 0.132 & 0.103 & 0.607 & $1.2 \pm 0.1$ & $1.2 \pm 0.1$ & $1.4 \pm 0.1$ & 0.028 & 0.016 & 0.039 \\
\hline
\end{tabular}


Table 2. Cont

\begin{tabular}{|c|c|c|c|c|c|c|c|c|c|c|c|c|c|c|c|c|c|c|}
\hline & \multicolumn{6}{|c|}{ KHEI } & \multicolumn{6}{|c|}{ aMED } & \multicolumn{6}{|c|}{ DASH } \\
\hline & T1 & $\mathrm{T} 2$ & T3 & $p$-Value & $\begin{array}{l}p \text {-Value } \\
\text { (T1 vs. } \\
\text { T3) }\end{array}$ & $\begin{array}{l}p \text {-Value } \\
\text { (T2 vs. } \\
\text { T3) }\end{array}$ & T1 & $\mathrm{T} 2$ & T3 & $p$-Value & $\begin{array}{l}p \text {-Value } \\
\text { (T1 vs. } \\
\text { T3) }\end{array}$ & $\begin{array}{c}p \text {-Value } \\
\text { (T2 vs. } \\
\text { T3) }\end{array}$ & T1 & $\mathrm{T} 2$ & $\mathrm{~T} 3$ & $p$-Value & $\begin{array}{l}p \text {-Value } \\
\text { (T1 vs. } \\
\text { T3) }\end{array}$ & $\begin{array}{c}p \text {-Value } \\
\text { (T2 vs. } \\
\text { T3) }\end{array}$ \\
\hline \multicolumn{19}{|l|}{ Women } \\
\hline$n$ & 655 & 656 & 655 & & & & 736 & 410 & 820 & & & & 633 & 694 & 639 & & & \\
\hline Low muscle strength ${ }^{2}, \mathrm{n}(\%)$ & $171(26.2)$ & 130 (19.3) & $87(14.0)$ & $<0.001$ & & & $200(27.3)$ & $86(20.5)$ & $102(12.6)$ & $<0.001$ & & & $158(24.4)$ & $151(22.4)$ & $79(12.5)$ & $<0.001$ & & \\
\hline Age, $y$ & $74.0 \pm 0.2$ & $72.8 \pm 0.2$ & $71.5 \pm 0.2$ & $<0.001$ & $<0.001$ & $<0.001$ & $74.0 \pm 0.2$ & $73.1 \pm 0.3$ & $71.5 \pm 0.2$ & $<0.001$ & $<0.001$ & $<0.001$ & $73.4 \pm 0.2$ & $73.1 \pm 0.2$ & $71.8 \pm 0.2$ & $<0.001$ & $<0.001$ & $<0.001$ \\
\hline $\begin{array}{c}\text { BMI, } \mathrm{kg} / \mathrm{m}^{2} \\
\text { Education } \mathrm{n}(\%)\end{array}$ & $24.4 \pm 0.2$ & $24.4 \pm 0.2$ & $24.5 \pm 0.1$ & 0.779 & 0.492 & 0.643 & $24.4 \pm 0.1$ & $24.5 \pm 0.2$ & $24.4 \pm 0.1$ & 0.965 & 0.896 & 0.874 & $24.4 \pm 0.2$ & $24.4 \pm 0.2$ & $24.5 \pm 0.1$ & 0.936 & 0.718 & 0.908 \\
\hline$<=$ Elementary school & $517(87.8)$ & $464(76.9)$ & $361(58.6)$ & $<0.001$ & & & $547(83.3)$ & 295 (78.7) & $500(63.8)$ & $<0.001$ & & & $478(83.9)$ & $465(72.7)$ & $399(66.1)$ & $<0.001$ & & \\
\hline$>$ Elementary school & $58(12.2)$ & $135(23.1)$ & 249 (41.4) & & & & $100(16.7)$ & $76(21.3)$ & $266(36.2)$ & & & & $86(16.1)$ & $160(27.3)$ & 196 (33.9) & & & \\
\hline No response & 80 & 57 & 45 & & & & 89 & 39 & 54 & & & & 69 & 69 & 44 & & & \\
\hline \multicolumn{19}{|l|}{ Smoking status, $\mathrm{n}(\%)$} \\
\hline $\begin{array}{l}\text { Nonsmoker } \\
\text { Noj }\end{array}$ & $537(89.8)$ & 579 (94.6) & $600(95.4)$ & 0.020 & & & $609(90.3)$ & 368 (94.5) & 739 (95.4) & 0.023 & & & $524(89.8)$ & $602(93.9)$ & $590(96.1)$ & 0.003 & & \\
\hline Ex-smoker & $34(5.4)$ & $19(3.4)$ & $18(2.9)$ & & & & $33(4.9)$ & $10(3.4)$ & $28(3.1)$ & & & & $29(5.0)$ & $27(4.2)$ & $15(2.4)$ & & & \\
\hline Current smoker & $20(4.8)$ & $11(2.1)$ & $12(1.8)$ & & & & $24(4.8)$ & $6(2.1)$ & $13(1.5)$ & & & & $22(5.2)$ & $12(1.9)$ & $9(1.5)$ & & & \\
\hline \multirow{2}{*}{\multicolumn{19}{|c|}{ Alcohol consumption, n (\%) }} \\
\hline & & & & & & & & & & & & & & & & & & \\
\hline Never & $380(62.6)$ & $387(64.0)$ & $373(57.8)$ & $<0.001$ & & & $439(65.0)$ & $241(62.8)$ & $460(57.7)$ & 0.018 & & & $361(60.8)$ & $396(61.1)$ & $383(62.3)$ & 0.145 & & \\
\hline$<1 \mathrm{drink} /$ month & 132 (22.7) & $169(27.7)$ & $189(30.5)$ & & & & $149(23.1)$ & $106(26.9)$ & 235 (30.7) & & & & $143(25.6)$ & $173(27.5)$ & $174(28.2)$ & & & \\
\hline $1 \mathrm{drink} / \mathrm{month}$ & $40(7.0)$ & $33(4.9)$ & $44(7.5)$ & & & & $43(6.6)$ & $24(6.0)$ & $50(6.5)$ & & & & $38(6.7)$ & $42(6.8)$ & $37(5.9)$ & & & \\
\hline 2-4 drink/month & $20(3.7)$ & $18(2.9)$ & $19(3.2)$ & & & & $19(2.5)$ & $9(2.4)$ & $29(4.3)$ & & & & $19(3.4)$ & $20(3.4)$ & $18(2.9)$ & & & \\
\hline$>=4 \mathrm{drink} / \mathrm{month}$ & $22(4.1)$ & $4(0.4)$ & $5(1.0)$ & & & & $18(2.8)$ & $7(1.9)$ & $6(0.8)$ & & & & $18(3.4)$ & $9(1.2)$ & $4(0.8)$ & & & \\
\hline \multirow{2}{*}{\multicolumn{19}{|c|}{$\begin{array}{c}\text { No response } \\
\text { Days of strength exercise (days/week), } n \text { (\%) }\end{array}$}} \\
\hline & & & & & & & & & & & & & & & & & & \\
\hline No & $542(93.5)$ & $546(92.8)$ & $516(83.9)$ & $<0.001$ & & & $608(93.2)$ & $338(91.9)$ & $658(86.1)$ & 0.002 & & & $528(93.4)$ & $565(90.4)$ & $511(86.2)$ & 0.009 & & \\
\hline 1 day/week & $4(1.0)$ & $7(1.0)$ & $13(2.0)$ & & & & $9(1.4)$ & $3(0.7)$ & 12 (1.5) & & & & $9(1.6)$ & $9(1.2)$ & $6(1.1)$ & & & \\
\hline 2 days/week & $9(1.5)$ & $11(1.9)$ & $9(1.4)$ & & & & $7(1.2)$ & $6(1.1)$ & $16(2.1)$ & & & & $9(1.5)$ & $10(1.6)$ & $10(1.6)$ & & & \\
\hline 3 days/week & $3(0.4)$ & $11(1.5)$ & $19(3.2)$ & & & & $6(0.7)$ & $3(0.7)$ & $24(3.1)$ & & & & $5(0.6)$ & $9(1.4)$ & $19(3.1)$ & & & \\
\hline 4 days/week & $4(0.7)$ & $2(0.3)$ & $14(2.3)$ & & & & $4(0.6)$ & $4(0.8)$ & $12(1.6)$ & & & & $3(0.4)$ & $9(1.4)$ & $8(1.4)$ & & & \\
\hline$>=5$ days/week & $16(3.0)$ & $21(2.8)$ & $42(7.3)$ & & & & $16(2.9)$ & $17(4.8)$ & $46(5.5)$ & & & & $12(2.5)$ & $24(4.0)$ & $43(6.6)$ & & & \\
\hline No response & 77 & 58 & 42 & & & & 86 & 39 & 52 & & & & 67 & 68 & 42 & & & \\
\hline \multicolumn{19}{|l|}{ Dietary supplement use, $n(\%)$} \\
\hline No & $393(57.8)$ & $338(50.3)$ & $256(37.9)$ & $<0.001$ & & & $441(58.3)$ & $196(43.5)$ & $350(42.3)$ & $<0.001$ & & & $362(55.9)$ & $348(48.5)$ & $277(41.4)$ & $<0.001$ & & \\
\hline Yes & $262(42.2)$ & $\begin{array}{l}318(49.7) \\
318\end{array}$ & $399(62.1)$ & & & & $295(41.7)$ & $214(56.5)$ & $470(57.7)$ & & & & $271(44.1)$ & $346(51.5)$ & $362(58.6)$ & & & \\
\hline Energy intake, kcal/d & $\begin{array}{c}1438.4 \pm \\
30.6\end{array}$ & $\begin{array}{c}1454.8 \pm \\
26.6\end{array}$ & $\begin{array}{l}1537.1 \pm \\
25.3\end{array}$ & 0.015 & 0.012 & 0.018 & $\begin{array}{l}1293.0 \pm \\
24.1\end{array}$ & $\begin{array}{c}1422.6 \pm \\
27.3\end{array}$ & $\begin{array}{c}1669.5 \pm \\
27.9\end{array}$ & $<0.001$ & $<0.001$ & $<0.001$ & $\begin{array}{l}1429.3 \pm \\
28.2\end{array}$ & $\begin{array}{l}1471.9 \pm \\
27.2\end{array}$ & $\begin{array}{c}1529.4 \pm \\
28.5\end{array}$ & 0.043 & 0.013 & 0.130 \\
\hline $\begin{array}{l}\text { Number of physician diagnosed } \\
\text { f }\end{array}$ & $1.7 \pm 0.1$ & $1.8 \pm 0.1$ & $1.9 \pm 0.1$ & 0.096 & 0.042 & 0.662 & $1.8 \pm 0.1$ & $1.8 \pm 0.1$ & $1.8 \pm 0.1$ & 0.825 & 0.946 & 0.554 & $1.7 \pm 0.1$ & $1.8 \pm 0.1$ & $1.9 \pm 0.1$ & 0.038 & 0.011 & 0.166 \\
\hline Age at menarche, $\mathrm{y}$ & $16.1 \pm 0.1$ & $15.5 \pm 0.1$ & $15.4 \pm 0.1$ & $<0.001$ & $<.001$ & 0.418 & $15.9 \pm 0.1$ & $15.8 \pm 0.1$ & $15.5 \pm 0.1$ & 0.005 & 0.002 & 0.018 & $16.0 \pm 0.1$ & $15.7 \pm 0.1$ & $15.4 \pm 0.1$ & $<0.001$ & $<0.001$ & 0.007 \\
\hline
\end{tabular}

1 Values are means \pm SDs unless otherwise specified. KNHANES, Korean National Health and Nutrition Examination Survey; KHEI, Korean Healthy Eating Index; aMED, alternate Mediterranean Diet; DASH, Dietary Approaches to Stop Hypertension; T, tertile, y, year. ${ }^{2}$ Low muscle strength was defined as the lower 20 th percentile of hand grip strength (men, 28.6 $\mathrm{kg}$; women, $16.4 \mathrm{~kg})$. 
Table 3. Correlation coefficients among diet quality indices for the KHEI, aMED, and DASH score of elderly men and women in the 2014-2016 KNAHNES 1.

\begin{tabular}{lcccccc}
\hline & \multicolumn{2}{c}{ KHEI } & \multicolumn{2}{c}{ aMED } & \multicolumn{2}{c}{ DASH } \\
\cline { 2 - 7 } & Men & Women & Men & Women & Men & Women \\
\hline KHEI & 1.00 & 1.00 & 0.38 & 0.43 & 0.53 & 0.55 \\
aMDS & 0.38 & 0.43 & 1.00 & 1.00 & 0.59 & 0.60 \\
DASH & 0.53 & 0.55 & 0.59 & 0.60 & 1.00 & 1.00 \\
\hline
\end{tabular}

${ }^{1}$ All $p<0.0001$. KNHANES, Korean National Health and Nutrition Examination Survey; KHEI, Korean Healthy Eating Index; aMED, alternate Mediterranean Diet; DASH, Dietary Approaches to Stop Hypertension.

Table 4 shows that across all indices, men and women in the 3rd tertile, compared with men and women in the 1st tertile had 32-53\% lower odds of low HGS. For both men and women, when comparing the 3rd and 1st tertile, the direction and magnitude of the adjusted ORs consistently indicated an inverse association for risk of low HGS. For example, the ORs for risk of low HGS were as follows: KHEI (OR: 0.57, 95\% CI: 0.35-0.91, p-trend = 0.015), aMED (OR: 0.64, 95\% CI: 0.44-0.93, $p$-trend $=0.014)$, and DASH (OR: 0.63, 95\% CI: 0.41-0.94, $p$-trend $=0.032)$ in men; and KHEI (OR: 0.68 , 95\% CI: 0.46-0.99, $p$-trend = 0.053), aMED (OR: 0.47, 95\% CI: 0.31-0.69, $p$-trend < 0.001), and DASH (OR: $0.52,95 \%$ CI: $0.34-0.79, p$-trend $=0.004$ ) in women.

\section{Discussion}

In this nationally representative cross-sectional study, we found that higher index scores for diet quality were associated with $32 \%-53 \%$ lower odds of low HGS in elderly Korean men and women, which were similar across all three diet quality indices-KHEI, aMED, and DASH. To our knowledge, this is the first study to identify that better overall diet quality may be associated with higher HGS in the elderly Korean population. Moreover, no previous literature studies have compared these specific indices within the same Korean population for the prevalence of low HGS.

The positive associations between diet quality and HGS are consistent with other recent (non-Korean) cross-sectional studies [15,24]. Fanelli et al. [15] found that adherence to the DASH eating pattern was positively associated with relative HGS in men and women in the USA. Barrea et al. [24] reported a positive association between adherence to the MED and HGS in a sample of active Italian senior women, stratified according to the HGS > cutoff-point of $20 \mathrm{~kg}$. Conversely, a cross-sectional study conducted in Italian elderly men and women [23] found no statistically significant associations between higher adherence to a MED and HGS, and a longitudinal study conducted in Canada [21] showed no significant association between diet quality measured using the Canadian Healthy Eating Index at baseline and the maintenance of three measures of muscle strength, including HGS.

Some studies [22,42] have shown that high adherence to a MED is significantly related to frailty but not to HGS, one of the criteria included in the definition of frailty. Bollwein et al. [42] found an association between a healthy diet, as measured using a MED score, and lower odds of frailty (defined as having three or more of the following five criteria: weight loss, feelings of exhaustion, poor HGS, low walking speed, or low physical activity). The association existed for low walking speed and low physical activity but not exhaustion and low HGS in elderly German individuals aged 75 years or older. Talegawkar et al. [22] reported that high adherence to a MED at baseline was associated with a lower risk of developing frailty (defined as having two or more of the following four criteria: feelings of exhaustion, poor HGS, low walking speed, or low physical activity) during a 6-year follow-up in a cohort of older, community-dwelling Italian men and women aged 65 years or older. However, when the authors looked at a high adherence to a MED and the relevance of each factor to frailty, diet was significantly related to low walking speed and low physical activity but not to feelings of exhaustion or poor HGS [22]. Therefore, the current evidence regarding the relationship between diet quality and HGS is inconsistent. 
Table 4. Multivariate odds ratios (ORs) and 95\% confidence intervals (CIs) for low muscle strength ${ }^{1}$ according to tertiles of diet quality indices for the KHEI, aMED, and DASH score among elderly men and women in the 2014-2016.

\begin{tabular}{|c|c|c|c|c|c|c|c|c|c|c|c|c|}
\hline & \multicolumn{6}{|c|}{ Men } & \multicolumn{6}{|c|}{ Women } \\
\hline & \multicolumn{2}{|c|}{ Unadjusted } & \multicolumn{2}{|c|}{ Model 1} & \multicolumn{2}{|c|}{ Model 2} & \multicolumn{2}{|c|}{ Unadjusted } & \multicolumn{2}{|c|}{ Model 1} & \multicolumn{2}{|c|}{ Model 2} \\
\hline & OR & $(95 \% \mathrm{CI})$ & OR & $(95 \% \mathrm{CI})$ & OR & $(95 \% \mathrm{CI})$ & OR & $(95 \% \mathrm{CI})$ & OR & $(95 \% \mathrm{CI})$ & OR & $(95 \% \mathrm{CI})$ \\
\hline \multicolumn{13}{|l|}{ KHEI } \\
\hline $\mathrm{T} 1$ & \multicolumn{2}{|c|}{1.00 (ref) } & \multicolumn{2}{|c|}{1.00 (ref) } & \multicolumn{2}{|c|}{1.00 (ref) } & \multicolumn{2}{|c|}{1.00 (ref) } & \multicolumn{2}{|c|}{1.00 (ref) } & \multicolumn{2}{|c|}{1.00 (ref) } \\
\hline $\mathrm{T} 2$ & 0.87 & $(0.637,1.178)$ & 1.12 & $(0.829,1.730)$ & 1.27 & $(0.878,1.837)$ & 0.67 & $(0.504,0.899)$ & 0.77 & $(0.536,1.106)$ & 0.75 & $(0.522,1.074)$ \\
\hline $\mathrm{T} 3$ & 0.47 & $(0.326,0.681)$ & 0.55 & $(0.344,0.875)$ & \multirow{2}{*}{\multicolumn{2}{|c|}{$0.015^{(0.35,, 0.919)}$}} & 0.46 & $(0.332,0.634)$ & 0.66 & $(0.456,0.967)$ & 0.68 & $(0.468,0.999)$ \\
\hline P for trend & \multicolumn{2}{|c|}{$<0.001$} & \multicolumn{2}{|c|}{0.008} & & & \multicolumn{2}{|c|}{$<0.001$} & \multicolumn{2}{|c|}{0.027} & \multicolumn{2}{|c|}{0.053} \\
\hline $\mathrm{T} 1$ & \multicolumn{2}{|c|}{1.00 (ref) } & \multicolumn{2}{|c|}{$1.00(\mathrm{ref})$} & \multicolumn{2}{|c|}{1.00 (ref) } & \multicolumn{2}{|c|}{$1.00(\mathrm{ref})$} & \multicolumn{2}{|c|}{1.00 (ref) } & \multicolumn{2}{|c|}{$1.00(\mathrm{ref})$} \\
\hline $\mathrm{T} 2$ & 0.68 & $(0.476,0.968)$ & 0.89 & $(0.594,1.343)$ & 0.91 & $(0.602,1.377)$ & 0.69 & $(0.486,0.972)$ & 0.74 & $(0.496,1.102)$ & 0.74 & $(0.499,1.110)$ \\
\hline T3 & 0.38 & $(0.275,0.517)$ & 0.59 & $(0.409,0.861)$ & 0.64 & $(0.440,0.933)$ & 0.38 & $(0.280,0.526)$ & 0.45 & $(0.308,0.668)$ & 0.47 & $(0.319,0.699)$ \\
\hline P for trend & \multicolumn{2}{|c|}{$<0.001$} & \multicolumn{2}{|c|}{0.005} & \multicolumn{2}{|c|}{0.014} & \multicolumn{2}{|c|}{$<0.001$} & \multicolumn{2}{|c|}{$<0.001$} & \multicolumn{2}{|c|}{$<0.001$} \\
\hline \multicolumn{13}{|l|}{ DASH } \\
\hline $\mathrm{T} 1$ & & (ref) & & (ref) & & (ref) & & (ref) & & (ref) & & (ref) \\
\hline $\mathrm{T} 2$ & 0.88 & $(0.658,1.186)$ & 0.78 & $(0.545,1.119)$ & 0.81 & $(0.560,1.158)$ & 0.84 & $(0.618,1.153)$ & 0.80 & $(0.546,1.176)$ & 0.82 & $(0.884,1.214)$ \\
\hline T3 & 0.66 & $(0.469,0.931)$ & 0.57 & $(0.377,0.859)$ & 0.63 & $(0.413,0.948)$ & 0.54 & $(0.386,0.763)$ & 0.51 & $(0.334,0.769)$ & 0.52 & $(0.343,0.796)$ \\
\hline$P$ for trend & & & & & & & & & & & & \\
\hline
\end{tabular}

${ }^{1}$ Low muscle strength was defined as the lower 20th percentile of hand grip strength (men, $28.6 \mathrm{~kg}$; women, $16.4 \mathrm{~kg}$ ). KNHANES, Korean National Health and Nutrition Examination Survey; KHEI, Korean Healthy Eating Index; aMED, alternate Mediterranean Diet; DASH, Dietary Approaches to Stop Hypertension; T, tertile; ref, reference. Model 1: adjusted for age, BMI, education, smoking, alcohol drinking, number of physician diagnosed chronic conditions, energy intake, and age at menarche (women only) Model 2: adjusted for the variables in model 1 , and physical activity 
Some studies have examined the relationship between diet quality and leg strength (knee extension), instead of HGS, as an indicator of muscle strength. A cross-sectional study of men and women aged 60 years or older in the USA [43] found that a higher total HEI 2005 score was associated with greater knee extension strength; however, this association was not statistically significant after adjustment for physical activity. Similarly, a longitudinal study of elderly Japanese women [44] found no significant association between diet quality (dietary variety) and knee extension strength. In contrast, a longitudinal study of Finnish elderly [25] showed that adherence to the NDS predicted greater HGS and knee extension strength 10 years later among women but not among men. The results of the study on leg strength and diet quality are also inconsistent. Therefore, a comprehensive study is needed in the future.

In our study, the exact mechanism of the relationship between all three diet quality indices-KHEI, aMED, and DASH - and HGS is not known, but several possible links could mediate the positive relationships between them. For example, elderly people with healthy dietary patterns are more likely to also have a healthy lifestyle, characteristics of which include participation in physical activity and regular exercise [45]. Such activity may help prevent age-related decreases in muscle mass or function [14,46]. Moreover, adherence to a high-quality diet, quantified using indices such as HEI, aMED, and DASH, has been shown to decrease the likelihood of obesity [47-49], a factor closely associated with decline in muscle strength and mass [50]. In addition, higher diet quality seems to be associated with antioxidant and anti-inflammatory responses. Adherence to KHEI, aMED, and DASH eating patterns, which are rich in antioxidant nutrients such as magnesium and vitamins $\mathrm{C}$ and $\mathrm{E}$, were associated with lower blood levels of inflammatory markers, such as $\mathrm{C}$-reactive protein and interleukin-6 [51]. In particular, a high score on the aMED indicates a higher intake of omega-3 fatty acids and monounsaturated fatty acids, which appear to be associated with anti-inflammatory effects [52]. Chronic low-grade systemic inflammation and oxidative stress can generate catabolism and increase protein turnover in skeletal muscle, reducing strength $[53,54]$, and can also increase the generation of reactive oxygen species, resulting in an overload of the antioxidant defense system [55].

We observed that across all indices, both men and women in the 3rd tertile were more likely to be more educated and physically active, have never smoked, and consume dietary supplements. This is consistent with previous studies [56-58] showing that people with higher diet quality are more likely to have higher levels of education and healthier lifestyles than those who have lower diet quality. We also observed that across all indices, men in the 3rd tertile had higher BMIs, compared to those in the lowest tertile, and women in the 3rd tertile had higher energy intakes when compared to those in the lowest tertile. Given that older adults are at an increased susceptibility of malnutrition due to age-associated changes in metabolism and physiology [59], our results demonstrate that increased BMI and energy intake may be favorable for the elderly.

In the present study, the relationship between diet quality indicators and HGS existed in both men and women. Similarly, a study of the elderly in the USA showed a relationship between diet quality and HGS in both men and women [15]. Some studies $[25,60,61]$ have indicated that the effects of diet quality index or dietary pattern on HGS might be different for men and women; however, the evidence was inconsistent. One of the cross-sectional studies [60] found that a healthier eating pattern was independently associated with higher HGS in women but not in men. The longitudinal study conducted on elderly Finnish people [25] reported that the healthy NDS predicts higher HGS 10 years later in senior women but not senior men. In a longitudinal study conducted in the UK [61], dietary patterns high in red meats, potato and gravy, or butter were associated with a lower HGS and a greater decline in HGS in men, whereas no association was observed in women. Since no other research on this topic has been conducted on Koreans, it is not possible to know whether the gender difference is dependent upon race. Further research is needed in the future.

One important limitation of this study was the inability to define causal associations with this dataset because of the cross-sectional study design. Further prospective studies are warranted to investigate the causal associations between diet quality or dietary pattern and HGS. Another limitation 
is that our dietary data was derived from a single 24-h dietary recall survey, which may provide an inaccurate estimate of a normal diet. However, the KNHANES report has shown that variations in data (3.9\% for energy, within 10\% for macro- and micro-nutrients) from a single day and 2-10 days of 24-h dietary recall were not significantly different [62]. Nevertheless, to the best of our knowledge, this is the first study identifying that better overall diet quality may be associated with higher HGS in the elderly Korean population, based on a nationally representative sample. This study has the potential to inform both policymakers and persons developing dietary guidelines about the role of diet quality or dietary patterns on the health of older adults.

\section{Conclusions}

We found that better overall diet quality may be associated with higher HGS in the elderly Korean population. Understanding the relationship between diet quality and HGS may offer opportunities for prevention and timely interventions. Future studies with larger sample sizes and prospective or interventional design are needed to further improve our knowledge about the association between diet quality or dietary patterns and HGS.

Author Contributions: O.K. and H.K. conceived and designed the study; H.K. performed the statistical analysis; H.K. wrote the paper; O.K. contributed to critical review of manuscript. All authors read and approved the final manuscript.

Funding: This research was funded by the Bio-synergy Research Project (NRF2012M3A9C4048761) of the Ministry of Science, ICT and Future Planning through the National Research Foundation and RP-Grant 2018 of Ewha Womans University, South Korea.

Conflicts of Interest: The authors declare no conflict of interest.

\section{References}

1. Morrison, S.; Newell, K.M. Aging, neuromuscular decline, and the change in physiological and behavioral complexity of upper-limb movement dynamics. J. Aging Res. 2012, 2012, 891218. [CrossRef] [PubMed]

2. Newman, A.B.; Kupelian, V.; Visser, M.; Simonsick, E.M.; Goodpaster, B.H.; Kritchevsky, S.B.; Tylavsky, F.A.; Rubin, S.M.; Harris, T.B. Strength, but not muscle mass, is associated with mortality in the health, aging and body composition study cohort. J. Gerontol. Ser. A Biol. Sci. Med. Sci. 2006, 61, 72-77. [CrossRef] [PubMed]

3. Vasconcelos, K.S.; Dias, J.M.; Bastone, A.C.; Vieira, R.A.; Andrade, A.C.; Perracini, M.R.; Guerra, R.O.; Dias, R.C. Handgrip strength cutoff points to identify mobility limitation in community-dwelling older people and associated factors. J. Nutr. Health Aging 2016, 20, 306-315. [CrossRef] [PubMed]

4. Springstroh, K.A.; Gal, N.J.; Ford, A.L.; Whiting, S.J.; Dahl, W.J. Evaluation of handgrip strength and nutritional risk of congregate nutrition program participants in Florida. J. Nutr. Gerontol. Geriatr. 2016, 35, 193-208. [CrossRef] [PubMed]

5. Bohannon, R.W.; Bear-Lehman, J.; Desrosiers, J.; Massy-Westropp, N.; Mathiowetz, V. Average grip strength: A meta-analysis of data obtained with a Jamar dynamometer from individuals 75 years or more of age. J. Geriatr. Phys. Ther. 2007, 30, 28-30. [CrossRef] [PubMed]

6. Izawa, K.P.; Watanabe, S.; Osada, N.; Kasahara, Y.; Yokoyama, H.; Hiraki, K.; Morio, Y.; Yoshioka, S.; Oka, K.; Omiya, K. Handgrip strength as a predictor of prognosis in Japanese patients with congestive heart failure. Eur. J. Cardiovasc. Prev. Rehabil. 2009, 16, 21-27. [CrossRef] [PubMed]

7. Pavasini, R.; Serenelli, M.; Celis-Morales, C.A.; Gray, S.R.; Izawa, K.P.; Watanabe, S.; Colin-Ramirez, E.; Castillo-Martínez, L.; Izumiya, Y.; Hanatani, S.; et al. Grip strength predicts cardiac adverse events in patients with cardiac disorders: An individual patient pooled meta-analysis. Heart 2019, 105, 834-841. [CrossRef]

8. Stenholm, S.; Tiainen, K.; Rantanen, T.; Sainio, P.; Heliövaara, M.; Impivaara, O.; Koskinen, S. Long-term determinants of muscle strength decline: Prospective evidence from the 22-year mini-Finland follow-up survey. J. Am. Geriatr. Soc. 2012, 60, 77-85. [CrossRef]

9. Cheung, C.L.; Nguyen, U.S.; Au, E.; Tan, K.C.; Kung, A.W. Association of handgrip strength with chronic diseases and multimorbidity: A cross-sectional study. Age 2013, 35, 929-941. [CrossRef] 
10. Rijk, J.M.; Roos, P.R.; Deckx, L.; van den Akker, M.; Buntinx, F. Prognostic value of handgrip strength in people aged 60 years and older: A systematic review and meta-analysis. Geriatr. Gerontol. Int. 2016, 16, 5-20. [CrossRef]

11. Abizanda, P.; Navarro, J.L.; Garcia-Tomas, M.I.; Paterna, G. Validity and usefulness of hand-held dynamometry for measuring muscle strength in community-dwelling older persons. Arch. Gerontol. Geriatr. 2012, 54, $21-27$. [CrossRef] [PubMed]

12. Norman, K.; Stobäus, N.; Reiß, J.; Schulzke, J.; Valentini, L.; Pirlich, M. Effect of sexual dimorphism on muscle strength in cachexia. J. Cachexia Sarcopenia Muscle 2012, 3, 111-116. [CrossRef] [PubMed]

13. Thorpe, R.J., Jr.; Simonsick, E.; Zonderman, A.; Evans, M.K. Association between race, poverty status and grip strength in middle to old age adults. Ethn. Dis. 2016, 26, 493-500. [CrossRef] [PubMed]

14. Tutkuviene, J.; Schiefenhovel, W. Laterality of handgrip strength: Age-and physical training-related changes in Lithuanian schoolchildren and conscripts. Ann. N. Y. Acad. Sci. 2013, 1288, 124-134. [CrossRef] [PubMed]

15. Kuczmarski, M.F.; Pohlig, R.T.; Shupe, E.S.; Zonderman, A.B.; Evans, M.K. Dietary protein intake and overall diet quality are associated with handgrip strength in African American and white adults. J. Nutr. Health Aging 2018, 22, 700-709. [CrossRef]

16. Houston, D.K.; Cesari, M.; Ferrucci, L.; Cherubini, A.; Maggio, D.; Bartali, B.; Johnson, M.A.; Schwartz, G.G.; Kritchevsky, S.B. Association between vitamin D status and physical performance: The InCHIANTI study. J. Gerontol. Ser. A Biol. Sci. Med. Sci. 2007, 62, 440-446. [CrossRef] [PubMed]

17. Lauretani, F.; Semba, R.D.; Bandinelli, S.; Dayhoff-Brannigan, M.; Giacomini, V.; Corsi, A.M.; Guralnik, J.M.; Ferrucci, L. Low plasma carotenoids and skeletal muscle strength decline over 6 years. J. Gerontol. Ser. A Biol. Sci. Med. Sci. 2008, 63, 376-383. [CrossRef]

18. Semba, R.D.; Blaum, C.; Guralnik, J.M.; Moncrief, D.T.; Ricks, M.O.; Fried, L.P. Carotenoid and vitamin E status are associated with indicators of sarcopenia among older women living in the community. Aging Clin. Exp. Res. 2003, 15, 482-487. [CrossRef]

19. Saito, K.; Yokoyama, T.; Yoshida, H.; Kim, H.; Shimada, H.; Yoshida, Y.; Iwasa, H.; Shimizu, Y.; Kondo, Y.; Handa, S.; et al. A significant relationship between plasma vitamin C concentration and physical performance among Japanese elderly women. J. Gerontol. Ser. A Biol. Sci. Med. Sci. 2012, 67, 295-301. [CrossRef]

20. Lauretani, F.; Semba, R.D.; Bandinelli, S.; Ray, A.L.; Guralnik, J.M.; Ferrucci, L. Association of low plasma selenium concentrations with poor muscle strength in older community-dwelling adults: The InCHIANTI Study. Am. J. Clin. Nutr. 2007, 86, 347-352. [CrossRef]

21. Rahi, B.; Morais, J.A.; Dionne, I.J.; Gaudreau, P.; Payette, H.; Shatenstein, B. The combined effects of diet quality and physical activity on maintenance of muscle strength among diabetic older adults from the NuAge cohort. Exp. Gerontol. 2014, 49, 40-46. [CrossRef] [PubMed]

22. Talegawkar, S.A.; Bandinelli, S.; Bandeen-Roche, K.; Chen, P.; Milaneschi, Y.; Tanaka, T.; Semba, R.D.; Guralnik, J.M.; Ferrucci, L. A higher adherence to a Mediterranean-style diet is inversely associated with the development of frailty in community-dwelling elderly men and women. J. Nutr. 2012, 142, 2161-2166. [CrossRef] [PubMed]

23. Fougère, B.; Mazzuco, S.; Spagnolo, P.; Guyonnet, S.; Vellas, B.; Cesari, M.; Gallucci, M. Association between the Mediterranean-style dietary pattern score and physical performance: Results from TRELONG study. $J$. Nutr. Health Aging 2016, 20, 415-419. [CrossRef] [PubMed]

24. Barrea, L.; Muscogiuri, G.; Di Somma, C.; Tramontano, G.; De Luca, V.; Illario, M.; Colao, A.; Savastano, S. Association between Mediterranean diet and hand grip strength in older adult women. Clin. Nutr. 2019, 38, 721-729. [CrossRef] [PubMed]

25. Perälä, M.M.; von Bonsdorff, M.B.; Männistö, S.; Salonen, M.K.; Simonen, M.; Kanerva, N.; Rantanen, T.; Pohjolainen, P.; Eriksson, J.G. The healthy Nordic diet predicts muscle strength 10 years later in old women, but not old men. Age Ageing 2017, 46, 588-594. [CrossRef] [PubMed]

26. Woo, E.K.; Han, C.; Jo, S.A.; Park, M.K.; Kim, S.; Kim, E.; Park, M.H.; Lee, J.; Jo, I. Morbidity and related factors among elderly people in South Korea: Results from the Ansan Geriatric (AGE) cohort study. BMC Public Health 2007, 7, 10. [CrossRef]

27. Jeong, G.W.; Kim, Y.J.; Park, S.; Kim, H.; Kwon, O. Associations of recommended food score and physical performance in Korean elderly. BMC Public Health 2019, 19, 128. [CrossRef] 
28. Lee, J.A. Relationship between grip strength and prevalence of hypertension in Korean adults: The Sixth Korea National Health and Nutrition Examination Survey (2015). Off. J. Korean Acad. Kinesiol. 2017, 19, $53-60$.

29. Lee, S.H.; Kim, S.J.; Han, Y.; Ryu, Y.J.; Lee, J.H.; Chang, J.H. Hand grip strength and chronic obstructive pulmonary disease in Korea: An analysis in KNHANES VI. Int. J. Chron. Obstruct. Pulmon. Dis. 2017, 12, 2313-2321. [CrossRef]

30. Oh, Y.H.; Moon, J.H.; Kong, M.H.; Oh, B.; Kim, H.J. The Association between hand grip strength and health-related quality of life in Korean adults. Korean J. Sports Med. 2017, 35, 103-111. [CrossRef]

31. Kwak, Y.; Kim, Y. Quality of life and subjective health status according to handgrip strength in the elderly: A cross-sectional study. Aging Ment. Health 2019, 23, 107-112. [CrossRef] [PubMed]

32. Ministry of Health and Welfare (KR). Korean National Health and Nutrition Examination Survey (KNHANES). Available online: http://knhanes.cdc.go.kr (accessed on 19 March 2009).

33. Yook, S.M.; Park, S.; Moon, H.K.; Kim, K.; Shim, J.E.; Hwang, J.Y. Development of Korean Healthy Eating Index for adults using the Korea National Health and Nutrition Examination Survey data. J. Nutr. Health 2015, 48, 419-428. [CrossRef]

34. Ministry of Health and Welfare (KR). Dietary Guidelines for Korean Adults; Ministry of Health and Welfare: Seoul, Korea, 8 December 2009. Available online: http://www.mw.go.kr/front_new/jb/sjb030301vw.jsp?PAR_ MENU_ID=03\&MENU_ID=0320\&CONT_SEQ=224044\&page=1 (accessed on 27 March 2014).

35. The Korean Nutrition Society. Dietary Reference Intake for Koreans, 1st ed.; The Korean Nutrition Society: Seoul, Korea, 2010.

36. Ministry of Health and Welfare (KR). Health Plan 2020; Ministry of Health and Welfare: Seoul, Korea, 2011. Available online: http://www.mw.go.kr/front_new/jb/sjb030301vw.jsp?PAR_MENU_ID=03\&MENU_ID= 0319\&CONT_SEQ=257824\&page=1 (accessed on 17 April 2014).

37. Trichopoulou, A.; Kouris-Blazos, A.; Wahlqvist, M.L.; Gnardellis, C.; Lagiou, P.; Polychronopoulos, E.; Vassilakou, T.; Liworth, L.; Trichopoulos, D. Diet and overall survival in elderly people. BMJ 1995, 311, 1457-1460. [CrossRef] [PubMed]

38. Fung, T.T.; McCullough, M.L.; Newby, P.K.; Manson, J.E.; Meigs, J.B.; Rifai, N.; Willett, W.; Hu, F.B. Diet-quality scores and plasma concentrations of markers of inflammation and endothelial dysfunction. Am. J. Clin. Nutr. 2005, 82, 163-173. [CrossRef] [PubMed]

39. Fung, T.; Chiuve, S.E.; Rexrode, K.M.; Hu, F.B. Adherence to a DASH-style diet and risk of coronary heart disease and stroke in women. Arch. Intern. Med. 2008, 168, 713-720. [CrossRef] [PubMed]

40. National Heart, Lung, and Blood Institute, US Department of Health and Human Services. Your Guide to Lowering Your Blood Pressure with DASH. Available online: http://www.nhlbi.nih.gov/health/public/heart/ hbp/dash/ (accessed on 1 March 2013).

41. Roberts, H.C.; Denison, H.J.; Martin, H.J.; Patel, H.P.; Syddall, H.; Cooper, C.; Sayer, A.A. A review of the measurement of grip strength in clinical and epidemiological studies: Towards a standardised approach. Age Ageing 2011, 40, 423-429. [CrossRef] [PubMed]

42. Bollwein, J.; Diekmann, R.; Kaiser, M.J.; Bauer, J.M.; Uter, W.; Sieber, C.C.; Volkert, D. Dietary quality is related to frailty in community-dwelling older adults. J. Gerontol. Ser. A Biol. Sci. Med. Sci. 2013, 68, 483-489. [CrossRef]

43. Xu, B.; Houston, D.K.; Locher, J.L.; Ellison, K.J.; Gropper, S.; Buys, D.R.; Zizza, C.A. Higher Healthy Eating Index-2005 scores are associated with better physical performance. J. Gerontol. Ser. A Biol. Sci. Med. Sci. 2012, 67, 93-99. [CrossRef]

44. Kojima, N.; Kim, M.; Saito, K.; Yoshida, H.; Yoshida, Y.; Hirano, H.; Obuchi, S.; Shimada, H.; Suzuki, T.; Kim, H. Lifestyle-Related Factors Contributing to Decline in Knee Extension Strength among Elderly Women: A Cross-Sectional and Longitudinal Cohort Study. PLoS ONE 2015, 10, e0132523. [CrossRef]

45. Reedy, J.; Krebs-Smith, S.M.; Miller, P.E.; Liese, A.D.; Kahle, L.L.; Park, Y.; Subar, A.F. Higher diet quality is associated with decreased risk of all-cause, cardiovascular disease, and cancer mortality among older adults. J. Nutr. 2014, 144, 881-889. [CrossRef]

46. Distefano, G.; Goodpaster, B.H. Effects of exercise and aging on skeletal muscle. Cold Spring Harb. Perspect. Med. 2018, 8, a029785. [CrossRef] [PubMed]

47. Drenowatz, C.; Shook, R.P.; Hand, G.A.; Hébert, J.R.; Blair, S.N. The independent association between diet quality and body composition. Sci. Rep. 2014, 4, 4928. [CrossRef] [PubMed] 
48. Agnoli, C.; Sieri, S.; Ricceri, F.; Giraudo, M.T.; Masala, G.; Assedi, M.; Panico, S.; Mattiello, A.; Tumino, R.; Giurdanella, M.C.; et al. Adherence to a Mediterranean diet and long-term changes in weight and waist circumference in the EPIC-Italy cohort. Nutr. Diabetes 2018, 8, 22. [CrossRef] [PubMed]

49. Soltani, S.; Shirani, F.; Chitsazi, M.J.; Salehi-Abargouei, A. The effect of dietary approaches to stop hypertension (DASH) diet on weight and body composition in adults: A systematic review and meta-analysis of randomized controlled clinical trials. Obes. Rev. 2016, 17, 442-454. [CrossRef] [PubMed]

50. Rolland, Y.; Czerwinski, S.; Abellan Van Kan, G.; Morley, J.E.; Cesari, M.; Onder, G.; Woo, J.; Baumgartner, R.; Pillard, F.; Boirie, Y.; et al. Sarcopenia: Its assessment, etiology, pathogenesis, consequences and future perspectives. J. Nutr. Health Aging 2008, 12, 433-450. [CrossRef] [PubMed]

51. Nowlin, S.Y.; Hammer, M.J.; D’Eramo Melkus, G. Diet, inflammation, and glycemic control in type 2 diabetes: An integrative review of the literature. J. Nutr. Metab. 2012, 2012, 542698. [CrossRef] [PubMed]

52. Casas, R.; Urpi-Sardà, M.; Sacanella, E.; Arranz, S.; Corella, D.; Castañer, O.; Lamuela-Raventós, R.M.; Salas-Salvadó, J.; Lapetra, J.; Portillo, M.P.; et al. Anti-Inflammatory Effects of the Mediterranean Diet in the Early and Late Stages of Atheroma Plaque Development. Mediat. Inflamm. 2017, 2017, 3674390. [CrossRef]

53. Welch, A.A. Nutritional influences on age-related skeletal muscle loss. Proc. Nutr. Soc. 2014, 73, 16-33. [CrossRef]

54. Otten, L.; Bosy-Westphal, A.; Ordemann, J.; Rothkegel, E.; Stobäus, N.; Elbelt, U.; Norman, K. Abdominal fat distribution differently affects muscle strength of the upper and lower extremities in women. Eur. J. Clin. Nutr. 2017, 71, 372-376. [CrossRef]

55. Le Lay, S.; Simard, G.; Martinez, M.C.; Andriantsitohaina, R. Oxidative stress and metabolic pathologies: From an adipocentric point of view. Oxidative Med. Cell. Longev. 2014, 2014, 908539. [CrossRef]

56. Gomes, A.P.; Soares, A.L.; Gonçalves, H. Low diet quality in older adults: A population-based study in southern Brazil. Cienc. Saude Coletiva 2016, 21, 3417-3428. [CrossRef] [PubMed]

57. de Freitas, T.I.; Previdelli, A.N.; Ferreira, M.P.D.; Marques, K.M.; Goulart, R.M.M.; de Aquino, R.D. Factors associated with diet quality of older adults. Rev. Nutr. 2017, 30, 297-306. [CrossRef]

58. de Souza Fernandes, D.P.; Duarte, M.S.L.; Pessoa, M.C.; Franceschini, S.D.C.C.; Ribeiro, A.Q. Evaluation of diet quality of the elderly and associated factors. Arch. Gerontol. Geriatr. 2017, 72, 174-180.

59. Drewnowski, A.; Evans, W.J. Nutrition, physical activity, and quality of life in older adults. J. Gerontol. Ser. A Biol. Sci. Med. Sci. 2001, 56 (Suppl. 2), 89-94. [CrossRef] [PubMed]

60. Robinson, S.M.; Jameson, K.A.; Batelaan, S.F.; Martin, H.J.; Syddall, H.E.; Dennison, E.M.; Cooper, C.; Sayer, A.A.; Hertfordshire Cohort Study Group. Diet and its relationship with grip strength in community-dwelling older men and women: The Hertfordshire cohort study. J. Am. Geriatr. Soc. 2008, 56, 84-90. [CrossRef] [PubMed]

61. Granic, A.; Jagger, C.; Davies, K.; Adamson, A.; Kirkwood, T.; Hill, T.R.; Siervo, M.; Mathers, J.C.; Sayer, A.A. Effect of Dietary Patterns on Muscle Strength and Physical Performance in the Very Old: Findings from the Newcastle 85+ Study. PLoS ONE 2016, 11, e0149699. [CrossRef] [PubMed]

62. Korea Centers for Disease Control and Prevention. Korea National Health and Nutrition Examination Survey (KNHANES) IV; Ministry of Health and Welfare: Seoul, Korea, 2010.

(C) 2019 by the authors. Licensee MDPI, Basel, Switzerland. This article is an open access article distributed under the terms and conditions of the Creative Commons Attribution (CC BY) license (http://creativecommons.org/licenses/by/4.0/). 